\title{
Research Square \\ An ancient motif unique for human STING, RGS12 and SARS-CoV-2 spike proteins
}

\section{Suleyman Aydin ( $\nabla$ saydin@anadolu.edu.tr)}

Anadolu University https://orcid.org/0000-0002-4052-8470

\section{Ayca Cakmak Aydin}

Yozgat Bozok University, Faculty of Medicine Dept. Medical Pharmacology https://orcid.org/00000003-1767-782X

\section{Biological Sciences - Article}

Keywords: T cell exhaustion, Cytokine Storm, Coagulation, Protein Motifs, Interferon Genes, Pathogenesis, Molecular Mimicry

Posted Date: January 12th, 2021

DOI: https://doi.org/10.21203/rs.3.rs-132824/v2

License: @ (i) This work is licensed under a Creative Commons Attribution 4.0 International License. Read Full License 
An ancient motif unique for human STING, RGS12 and SARS-CoV-2 spike proteins.

The coronavirus named as SARS-CoV-2 is the cause of the COVID-19 pandemic and

4 spreading rapidly ${ }^{1}$. It is a pneumonia outbreak ${ }^{2}$ with $\mathbf{T}$ cell exhaustion, cytokine storm and coagulation $^{3,4}$. Short motifs on proteins play important roles on protein-protein interactions ${ }^{5,6}$.

6 We hypothetized role of molecular mimicry of small-xxx-small motifs for the spike protein of SARS-CoV-2. Here we show that a unique and evolutionary conserved motif is found only on

8 the spike protein of SARS-CoV-2 and stimulator of interferon genes (STING) proteins. Surprisingly we could not find this motif on any other protein of any living form. We found a

10 similar, but not identical motif mimicry for the spike and regulator of G protein signaling 12 (RGS12), C1QT4 and also for proteins of Archaea and beta-lactamase enzymes of bacteria

12 including Mycobacterium tuberculosis. STING proteins have roles on coagulation, $T$ cell exhaustion, cytokine release $^{1-3}$ and RGS12 on inflammation ${ }^{7}$. In contrast to cGAS-STING

14 pathway $^{8}$, the motif mimicry indicated a direct interaction between spike and STING proteins suggesting the importance of STING, RGS12 and C1QT4 on the pathogenesis of COVID-19.

16 To our surprise, the molecular mimicry showed that beta-lactamase inhibitors may be effective against SARS-CoV-2. The motif is unique, as found on Archaea and Cnidaria it is evolutionary old but a new target and mechanism for the COVID-19. 


\section{Main}

22 COVID-19 pandemic is caused by the beta-coronavirus named as severe acute respiratory syndrome coronavirus (SARS-CoV-2) ${ }^{1-3}$. The number of cases are over seventy millions and the deaths are

24 more than one and a half million ${ }^{9}$. The COVID-19 is described as unknown pneumonia with gastrointestinal, cardiovascular, immunological and neurological complications. The most serious

26 complication of COVID-19 is hypoxemia due to the respiratory failure and many patients die from acute respiratory distress syndrome $(\mathrm{ARDS})^{1-3}$. Venous and arterial thrombosis is very common in

28 COVID-19 playing role on multisystem organ dysfunction. Thrombotic abnormalities and cardiovascular complications lead to ischemic stroke, myocardial infarction and venous

30 thromboembolism playing role on multisystem organ dysfunction in COVID-19 ${ }^{10}$. The pathophysiology is not fully defined but the spike protein of the virus plays role for entering the

32 host cells $\mathbf{s}^{1,3,11}$. There is no proven treatment for the COVID-19 ${ }^{12}$. The angiotensin converting enzyme type 2 (ACE2) was found as the main receptor for the spike protein ${ }^{1,11}$. ACE2 is absent on T

34 cells $^{13}$ and the interaction between ACE2 and the spike protein is not sufficient for explaining the mechanism(s) of coagulation and cytokine storms of the COVID-19.

36 GxxxG motifs are one of the small-xxx-small short linear motifs which have important role on the protein-protein interactions $s^{5,6}$ including virus proteins ${ }^{14}$. These motifs play role on the molecular

38 mimicry and evolutionary arms race $^{15}$. There are controversial results on the role of the GxxxG motif for the spike protein of the coronavirus ${ }^{16,17}$. The aim of our study was to investigate the

40 GxxxG (GG4) motifs on the spike protein of the SARS-CoV-2 using bioinformatical methods.

\section{\#1. Unique and evolutionary conserved motif}

42 There were more than one GG4 motifs on the spike protein but one of them was rich in aromatic amino acids. The first amino acid of the motif was alanine but not glycine. We named this small-

44 xxx-small motif as "semi-GG4 motif" which showed motif similarity with the stimulator of interferon genes (STING) proteins (Fig. 1). A molecular mimicry is present for the STING proteins 46 and spike protein of SARS-CoV-2 which can be shown as a single formula: [AS]YY[FIV]GYL 
(Fig. 1). The presence of this motif on the SPIKE proteins of many species including Nematostella

48 vectensis showed us the motif is evolutionary conserved on STING proteins since Cnidarians (Figs. 1,2 Extended Data Fig. 1). We were surprised to see that this motif mimicry is unique for the

50 SARS-CoV-2 and STING proteins (Fig. 1) because our search for this motif on the UniProt surprisingly showed that it is not found on any other protein.

\section{2 \#2. The motif and other coronaviruses}

A similar aromatic amino acid-rich semi-GG4 was found on the spike proteins of all coronaviruses

54 with a common motif: [ASL][YGKN][FYNSR][FYSVIL]G[FY][LC] (Extended Data Fig. 1).

\section{\#3. The spike group of the $21^{\text {st }}$ century}

56 Cluster analysis of the spike proteins of coronaviruses isolated from humans showed us that they can be classified as 3 subgroups. We named them as first (1s), second (2s) and third spike group

58 (3s). There are only three members of the third spike group (3s): MERS, SARS (SARS-CoV) and SARS2 (SARS-CoV-2) which are the causes of the serious infections of the $21^{\text {st }}$ century $\left(21^{\text {st }}\right.$

60 century group) (Fig. 2). The Venn diagram showed us that these pathogens of the $21^{\text {st }}$ century make up a separate group of the human beta-coronavirus spike proteins (Fig. 3).

62 The clinical differences between MERS, SARS-CoV and SARS-CoV-2 are known ${ }^{18}$ and a similar difference between the small-xxx-small motifs of these three spike proteins can be described in

64 terms of their aromatic amino acids. There is only one aromatic amino acid which is phenylalanine (Phe) on the motif of MERS and three aromatic amino acids (2 Tyr +1 Phe) on SARS-CoV but the three aromatic amino acids on SARS-CoV-2 are all tyrosine (Tyr) (Fig. 2).

\section{\#4. Tyrosine}

68 Tyrosine is important on the structure and function of proteins ${ }^{19,20}$. The amount of aromatic amino acids and Tyr content of the semi-GG4 motif of the spike proteins of the 3 s subgroup is parallel

70 with their pathogenic potentials: Number of aromatic amino acids as Tyr are highest on motif of the SARS-CoV-2 (Fig. 2) which is more contagious than others ${ }^{3}$.

\section{2 \#5. Location of the motif}


The amino acid numbers of the unique semi-GG4 motif are 264-270 on the N-terminal domain

74 (NTD) of the spike protein (accession number: P0DTC2) (Figs. 4a,b). NTD was reported as another binding region of SARS-CoV-2 $2^{21-23}$. The unique motif is also the binding site of the endogenous

76 ligand, cyclic-di-GMP of the STING protein $^{24}$.

\section{\#6. GYL triplet}

78 The GYL triplet of the unique motif is found on the spike proteins of SARS-CoV and SARS-CoV-2 but not on MERS (Fig. 2) suggesting a possible role of the GYL triplet on the different pathogenic

80 properties of MERS, SARS-CoV and SARS-CoV-2. IGY motif was also reported on the secreted toxic proteins of fungi ${ }^{25}$ which is found inside the unique motif indicating the role of evolutionary

82 mechanisms (Fig. 3).

\section{\#7. Aromatic cage}

84 Tyrosine as the $266^{\text {th }}$ amino acid $\left(\mathrm{Y}^{266}\right)$ is found only on the SARS-CoV-2 but not on SARS-CoV and MERS (Figs. 2, 4b). There is hydrophobic contact between $\mathrm{Y}^{266}$ and $\mathrm{W}^{64}$ and they make an

86 aromatic cage. $\mathrm{Y}^{266}$ is attached to the $\mathrm{R}^{214}$ and $\mathrm{A}^{93}$ of the neighbouring beta-sheets (Fig. 4c) contributing to a more stabilized structure as reported for some other proteins ${ }^{26,27}$. There is another

88 aromatic cage just nearby the $\mathrm{Y}^{266}$ (Fig. 4d) showing that the unique motif is found in an aromatic cage-rich area. This structure is found only on the spike of SARS-CoV-2 but not on other members

90 of the 3s group because they (SARS-CoV and MERS) do not have $\mathrm{Y}^{266}$ and $\mathrm{W}^{64}$ amino acids (Figs. 2, 5c). The unique motif is rich in aromatic amino acids and aromatic cages (Figs. 4c,d). Aromatic

92 cages usually capture positively charged molecules and amino acids like lysine ${ }^{26}$ but there is no data on this aromatic cage of the spike protein and we do not know the kind of role on the virus-host

94 relationships.

\section{\#8. STING protein}

96 Free DNA in the cytoplasm is abnormal and it starts the STING signaling. Intracellular genomic structures including viruses are sensed by the cyclic GMP synthase (cGAS) producing cyclic

98 dinucleotides like c-di-GMP which activate STING proteins ${ }^{28}$. Activated STING is important in 
autophagy $^{29}$, cytokine release ${ }^{1}$, coagulation ${ }^{30}$, obesity ${ }^{31,32}$ and old age ${ }^{33}$. These are among the

100 symptoms of COVID-19, which are all effected by the STING proteins. The unique motif is the cdi-GMP binding site on the STING protein ${ }^{24}$ showing the presence of a molecular mimicry enabling

102 a direct interaction between the STING and the spike proteins of SARS-CoV-2 (Figs. 1,2). The cdi-GMP binding site plays role on the direct interaction between STING and the spike proteins, as a

104 different mechanism from the cyclic guanosine monophosphate-adenosine monophosphate synthase-stimulator of interferon genes (cGAS-STING) pathway. This direct interaction, in addition

106 to the cGAS-STING pathway, will result with hyperactivation of the STING proteins. STING activation plays role on vascular and pulmonary pathologies ${ }^{30}$ and it is a major player for the

108 induction of neutrophil extracellular $\operatorname{traps}^{34}$ contributing to the immunothrombosis ${ }^{35}$. Activated STING proteins also have interferon-independent actions leading to T cell death ${ }^{36}$, but STING null

110 cells and organisms are highly susceptible to infections of viruses, bacteria and intracellular parasites like Plasmodium ${ }^{37}$ showing importance of the balance of the functions of STING

112 proteins $^{38}$. The unique motif shows us one of the mechanisms of hyperstimulation of STING proteins by the spike protein of SARS-CoV-2 leading to hyperinflammation, coagulation, $\mathrm{T}$ cell

114 exhaustion and high levels of neutrophil extracellular traps of COVID-19 and also responsible for the enhanced actions of COVID-19 on obese ${ }^{32}$ and the old age ${ }^{1}$.

\section{6 \#9. RGS12}

We found another small-xxx-small (semi-GG4) motif for the spike protein of SARS-CoV-2 and regulator of G protein signaling 12 (RGS12) proteins. It is similar, but not identical to the unique motif for the STING proteins which can be written as: A[MY][VIY]VGYL (Figs. 5a,b). We

120 searched for this motif and found that it is also unique and found only on the RGS12 and spike protein of SARS-CoV-2. RGS12 was recently reported to play a key role on inflammatory

122 reactions $^{7}$ suggesting a significant contribution to the pathogenesis of COVID-19. Our results do not indicate any role for the STING and RGS12 proteins on pain, anosmia, ageusia, sex differences 124 or the impact of air pollution on the COVID-19. 


\section{\#10. TRPM ion channels}

126 A surprising motif similarity between the spike protein of SARS-CoV-2 and a group of TRPM ion channels (TRPM1-TRPM4) is another example of molecular mimicry which we did not investigate

128 further because it was very different than the unique motif (Extended Data Fig. 2).

\section{\#11. Mycobacterium tuberculosis}

130 RGS12 and STING proteins are not specific for the respiratory system but the main pathogenic actions of Mycobacterium tuberculosis, Mycoplasma pneumonia and COVID-19 are on the

132 respiratory system. Some of the proteins of the well known pathogenic bacteria of the pulmonary infections including M. pneumonia, M. tuberculosis, Klebsiella and Yersinia species exhibit a motif

134 similarity to the unique motif. Their motifs are similar, but not identical to the unique motif and also they are poor in aromatic amino acids (Figs. 1-5, Extended Data Figs. 1,3). Tuberculosis/COVID-19

136 co-infections are reported which may converge in a "perfect storm" ${ }^{39}$. This motif similarity and the molecular mimicry may help us understand the interaction between tuberculosis and COVID-19.

\#12. C1QT4

It was also very surprising for us to find a motif very similar (but not identical) to the unique motif

140 for the beta-lactamase enzymes of M. tuberculosis and the STING proteins (Extended Data Fig. 4) and also for C1q tumor necrosis factor-related protein 4 (FASTA name is C1QT4) (Extended Data

142 Fig. 4a). The motif similarity between the $M$. tuberculosis beta-lactamase and C1QT4 was high compared to the STING proteins (Extended Data Fig. 4b). High levels of IL-6 is one of the severity

144 predictors in COVID-1940. C1QT4 is one the major IL-6 elevating mechanisms and plays role on viral infections ${ }^{41}$ indicating the role of C1QT4 on COVID-19 and supporting our results which was 146 not reported for COVID-19.

\section{\#13. Archaea and evolution}

148 It was interesting to find the same unique motif similarity between the spike proteins, ribosomal protein of Methanosprillum hungatei and membrane protein of Methanococcus maripaludis 150 (Extended Data Fig. 4c). These prokaryotes are members of anaerobic methanogen Archaea 
(Extended Data Fig. 4c) $)^{42-43}$ showing evolutionary relationships.

A small motif and a molecular mimicry enabling interactions of so many proteins, most if not all,

154 are involved in inflammatory reactions shows that the motif is short (only 7 amino acids) but not functionally so simple. If the sequence of the motif is [AS]YY[FIV]GYL, it is unique for STINGS 156 and the spike protein of the SARS-CoV-2 but the motif [AS]xxxGYL is found on many proteins including beta-lactamase, C1QT4, RGS12 and on the proteins of Archaea suggesting that the motif

158 is a member of "hub motifs"44 with many other features awaiting to be discovered.

The motif is an evolutionary conserved "hub motif" and possibly the STING protein is a "hub 160 protein"44.

\section{\#15. Beta-lactamase}

162 There was a second motif for the beta-lactamase and the spike of SARS-CoV-2. This second motif similarity between beta-lactamase and the spike was adjacent to the unique motif (Extended Data

164 Fig. 4d) suggesting an unusual interaction for beta-lactamase and the spike proteins. Based on this surprising molecular mimicry, we suggest that the classical beta-lactamase inhibitors are expected

166 to inhibit some of the pathological effects of COVID-19. There is no proven drug for the COVID$19^{45}$ and based on our results (Extended Data Fig. 4), beta-lactamase inhibitors are expected to be

168 effective which may at least reduce IL-6 levels. Beta-lactamase inhibitors can be applied to the patients without any delay.

\section{\#17. Molecular mimicry, evolution, unique motif and beta-lactamase}

Importance of the GxxxG motif was reported on the SARS-CoV-2 proteins ${ }^{46}$. Mimicry and

172 molecular mimicry are among the methods of the evolutionary arms race ${ }^{47-49}$ and mimicry was proposed as a mechanism to explain multi-organ damage in COVID-19 ${ }^{50}$.

174 The aim of our study was not to investigate the interactions and roles of STING, RGS12, C1QT4 proteins or beta-lactamase enzymes on COVID-19, but the unique motif led us to these proteins and

176 to our surprise, to the beta-lactamase inhibitors. Our results show the importance and presence of 
the evolutionary conserved motif mimicry.

178 There may be additional unique motifs on the proteins of SARS-CoV-2 which can help us to explain the unknowns of the COVID-19 and find effective medicines. The evolutionary conserved

180 molecular mimicry of the unique motif shows us that beta-lactamase inhibitors may be used against COVID-19. Role of the STING, RGS12, C1QT4 proteins and the unique motif on the COVID-19

182 are new for us but they are ancient which are found on proteins of Anthozoa and Archaea.

\section{References}

01. Hu, B., Guo, H., Zhou, P. \& Shi, Z-L. Characteristics of SARS-CoV-2 and COVID-19. Nat.

Rev. Microbiol. 6, 1-14 (2020).

02. Zhou, P. et al. A pneumonia outbreak associated with a new coronavirus of probable bat origin.

188 Nature 579, 270-273 (2020).

03. Tang, D., Comish, P. \& Kang, R. The hallmarks of COVID-19 disease. PLoS Pathog 16, 190 e1008536 (2020).

04. Moon, C. Fighting COVID-19 exhausts T cells. Nat Rev Immunol. 20, 277 (2020).

192 05. Lock, A. et al. One motif to bind them: A small-XXX-small motif affects transmembrane domain 1 oligomerization, function, localization and cross-talk between two yeast GPCRs. Biochim

194 Biophys Acta 1838, 3036-3051 (2014).

06. Teese, M.G. \& Langosch, D. Role of GxxxG motifs in transmembrane domain interactions.

Biochemistry 54, 5125-5135 (2015).

07. Yuan, G. et al. RGS12 is a novel critical NF-kB activator in inflammatory arthritis. iScience 23, $198101072(2020)$.

08. Wan, D., Jiang, W. \& Hao, J. Research advances in how the cGAS-STING pathway controls the cellular inflammatory response. Front Immunol. 11, 615 (2020).

09. World Health Organization WHO coronavirus disease (COVID-19) Dashboard. 202 https://covid19.who.int. 
10. Piazza, G \& Morrow D.A. Diagnosis, management, and pathophysiology of arterial and venous

204 thrombosis in COVID-19. JAMA doi:10.1001/jama.2020.23422 (2020).

11. Liu, L. et al. Potent neutralizing antibodies against multiple epitopes on SARS-CoV-2 spike.

206 Nature 584, 450-456 (2020).

12. Kim, P.S., Read, S.W. \& Fauci, A.S. Therapy for early COVID-19: A critical need. JAMA 208 doi:10.1001/jama.2020.22813 (2020).

13. Hamming, I. et al. Tissue distribution of ACE2 protein, the functional receptor for SARS 210 coronavirus. A first step in understanding SARS pathogenesis. J Pathol. 203, 631-637 (2004).

14. Bronnimann M.P., Chapman, J.A., Park, C.K. \& Campos, S.K. A transmembrane domain and

212 GxxxG motifs within L2 are essential for Papillomavirus infection. J Virol. 87, 464-473 (2013).

15. Guo, H. et al. Evolutionary arms race between virus and host drives genetic diversity in bat

214 severe acute respiratory syndrome-related coronavirus spike genes. J Virol. 94, 00902-20 (2020).

16. Arbely, E., Granot, Z., Kass, I., Orly, J. \& Arkin, I.T. A trimerizing GxxxG motif is uniquely

216 inserted in the severe acute respiratory syndrome (SARS) coronavirus spike protein transmembrane domain. Biochemistry 45, 11349 (2006).

218 17. Corver, J., Broer, R., van Kasteren, P. \& Spaan, W. GxxxG motif of severe acute respiratory syndrome coronavirus spike glycoprotein transmembrane domain is not involved in trimerization

220 and is not important for entry. J. Virol. 81, 8352-8355 (2007).

18. Zhang, Y-Y., Li, B-R. \& Ning, B-T. The comparative immunological characteristics of SARS-

222 CoV, MERS-CoV, and SARS-CoV-2 coronavirus infections. Front Immunol. 11, 2033 (2020).

19. Stenberg, G., Abdalla, A-M. \& Mannervik, B. Tyrosine 50 is at the subunit interface of dimeric

224 human glutathione transferase P1-1 is a structural key residue for mudulating protein stability and catalytic function. Biochim Biophys Res Commun. 271, 59-63 (2000).

226 20. Yao, J. \& Gillam, S. A single-amino-acid substitution of a tyrosine residue in the Rubella virus E1 cytoplasmic domain blocks virus release. J Virol. 74, 3029-3036 (2000).

228 21. Hulswit, R.J.G. et al. Human coronaviruses OC43 and HKU1 bind to 9- O-acetylated sialic 
acids via a conserved receptor-binding site in spike protein domain A. Proc Natl Acad Sci U S A 230 116, 2681-2690 (2019).

22. Behloul, N., Baha, S., Shi, R. \& Meng, J. Role of the GTNGTKR motif in the N-terminal

232 receptor-binding domain of the SARS-CoV-2 spike protein. Virus Res. 286, 198058 (2020).

23. Seyran, M. et al. The structural basis of accelerated host cell entry by SARS-CoV-2. FEBS J. 234 doi: 10.1111/febs.15651 (2020).

24. Huang, Y-H., Liu, X-Y., Du, X-X., Jiang, Z-F. \& Su, X-D. The structural basis for the sensing 236 and binding of cyclic di-GMP by STING. Nat Struct Mol Biol. 19, 728-730 (2012).

25. Cheng, Q. et al. Discovery of a novel small secreted protein family with conserved N-terminal

238 IGY motif in Dikarya fungi. BMC Genomics 15, 1151 (2014).

26. Cecchini, M. \& Changeux, J.P. The nicotinic acetylcholine receptor and its prokaryotic

240 homologues: Structure, conformational transitions and allosteric modulation. Neuropharmacology 96, 137-149 (2015).

242 27. Nirthanan, S. Snake three-finger alpha-neurotoxins and nicotinic acetylcholine receptors: molecules, mechanisms and medicine. Biochem. Pharmacol. 181, 114168 (2020).

244 28. Li, Z. et al. When STING meets viruses: Sensing, trafficking and response. Front Immunol. 11, 2064 (2020).

246 29. Gui, X. et al. Autophagy induction via STING trafficking is a primordial function of the cGAS pathway. Nature, 567, 262-266 (2019).

248 30. Liu, Y. et al. Activated STING in a vascular and pulmonary syndrome. N. Eng. J. Med. 371, 507-518 (2014).

250 31. Mao, Y. et al. STING-IRF3 triggers endothelial inflammation in response to free fatty acidinduced mitochondrial damage in diet-induced obesity. Arterioscler Thromb Vasc Biol. 37, 920-929 252 (2017).

32. Sattar, N., McInnes, I.B \& McMurray, J.J.V. Obesity is a risk factor for severe COVID-19 254 infection: Multiple potential mechanisms. Circulation 142, 4-6 (2020). 
33. Zhong, W. et. al. Aging aggravated liver ischemia and reperfusion injury by promoting STING-

256 mediated NLRP3 activation in macrophages. Aging Cell 19, e13186 (2020).

34. Veras, F.P. et al. SARS-CoV-2-triggered neutrophil extracellular traps mediate COVID-19 258 pathology J Exp Med. 217, e20201129 (2020).

35. Middleton, E.A. et al. Neutrophil extracellular traps contribute to immunothrombosis in COVID-19 acute respiratory distress syndrome. Blood 136, 1169-1179 (2020).

36. Wu, J., Dobbs, N., Yang, K. \& Yan, N. Interferon-independent activities of mammalian STING

262 mediate antiviral response and tumor immune evasion. Immunity 53, 115-126 (2020).

37. Bryant, C.E. et al. International Union of Basic and Clinical Pharmacology. XCVI. Pattern

264 Recognition Receptors in Health and Disease. Pharmacol Rev. 67, 462-504 (2015).

38. Landman, S.L., Ressing, M.E. \& van der Veen, A.G. Balancing STING in antimicrobial defense 266 and autoinflammation. Cytokine Growth Factor Rev. 55, 1-14 (2020).

39. Mousquer, G.T., Peres, A. \& Fiegenbaum, M. Pathology of TB/COVID-19 co-infection: The 268 phantom menace. Tuberculosis 126, 102020 (2020).

40. Han, H. et al. Profiling serum cytokines in COVID-19 patients reveals IL-6 and IL-10 are 270 disease severity predictors. Emerg. Microbes Infect. 9, 1123-1130 (2020).

41. $\mathrm{Xu}, \mathrm{W}$. et al. $\mathrm{C} 1 \mathrm{Q} / \mathrm{TNF}-$ related protein 4 expression correlates with herpes simplex encephalitis 272 progression. Ann Transl Med. 7, 235 (2019).

42. Hendrickson, E.L. et al. Complete genome sequence of the genetically tractable

274 hydrogenotrophic methanogen Methanococcus maripaludis. J Bacteriol 186, 6956-6969 (2004).

43. Gunsalus, R.P. et al. Complete genome sequence of Methanospirillum hungatei type strain JF1.

276 Stand Genomic Sci. 11, 2 (2016).

44. Johnson, M.E. \& Hummer, G. Evolutionary Pressure on the Topology of Protein Interface 278 Interaction Networks. J. Phys. Chem. B 117, 10.1021/jp402944e (2013).

45. Vijayvargiya, P. et al. Treatment considerations for COVID-19: A critical review of the 280 evidence (or lack thereof). Mayo Clin Proc. 95, 1454-1466 (2020). 
46. Ionescu, J. An overview of the crystallized structures of the SARS-CoV-2. Protein J. doi:

47. Chemes, L.B., de Prat-Gay, G.\& Sanches, I.E. Convergent evolution and mimicry of protein 284 linear motifs in host-pathogen interactions. Curr Opin Struct Biol. 32, 91-101 (2015).

48. Elde, N.C. \& Malik, H.S. The Evolutionary conundrum of pathogen mimicry. Nat Rev 286 Microbiol. 7, 787-797 (2009).

49. Guo, H. et al. Evolutionary arms race between virus and host drives genetic diversity in bat 288 severe acute respiratory syndrome-related coronavirus spike genes. J. Virol. 94, 00902-20 (2020).

50. Angileri, F. et al. Molecular mimicry may explain multi-organ damage in COVID-19. 290 Autoimmun Rev. 19, 102591 (2020).

51. Larkin, M.A. et al. Clustal W and Clustal X version 2.0. Bioinformatics 23, 2947-2948 (2007).

292 52. R Core Team (2019). R: A language and environment for statistical computing. R Foundation for Statistical Computing, Vienna, Austria. URL http://www.R-project.org/.

294 53. Rose, A.S. et al. NGL viewer: web-based molecular graphics for large complexes. Bioinformatics 34, 3755-3758 (2018). 


\section{Legends of the Figures}

298 Fig. 1: Alignment of the spike protein of SARS-CoV-2 and the STING proteins. The motif similarity ([AS]xxxGYL) is shaded. Black square represents the amino acids Ala and Ser [AS].

Fig. 2: Dendrogram of the phylogenetic relationships of the STING and the spike proteins of human

302 beta-coronaviruses based on alignment of amino acid sequences generated by Clustal Omega and hierarchical cluster analysis using $\mathrm{R}$. ' $\mathrm{x}$ ' denotes any amino acid. The first small amino acid in the small-xxx-small motifs are Ala(A), Leu(L) or Ser(S) and the other small amino acid is $\operatorname{Gly}(\mathrm{G})$ making the semi-GG4 motif. $1 \mathrm{~s}=$ First group, $2 \mathrm{~s}=$ Second group, $3 \mathrm{~s}=$ Third group. Members of the

$3063 \mathrm{~s}$ are causes of the viral outbreaks of the $21^{\text {st }}$ century and the ongoing pandemic of COVID-19.

308 Fig. 3: Venn diagram of the STING and the spike proteins of human beta-coronaviruses showing the $3 \mathrm{~s}$ as a distinct group as if it is evolving towards a new group of beta-coronavirus. The same

310 STING and the spike proteins shown in Fig. 2 were used for this Venn diagram.

312 Fig. 4: The unique motif is, (A) on the NTD of the spike protein marked with stars, (B) located on one of the beta-sheets with a finger like loop extending outside, (C) there is a hydrogen bond

314 between the $\mathrm{R}^{214}$ of the neighbour beta-sheet and $\mathrm{Y}^{266}$ which is found only on the SARS-CoV-2 member of the 3s group. $\mathrm{Y}^{266}$ makes an aromatic cage with the $\mathrm{W}^{64}$ which is found only on SARS-

$316 \mathrm{CoV}-2$, (D) another aromatic cage around $\mathrm{A}^{93}$, the unique motif is surrounded with aromatic cages.

318 Fig. 5: Motif similarity (A) for the STING, RGS12 and the spike proteins of SARS-CoV and SARS-CoV-2, (B) for the RGS12 and the spike protein of SARS-CoV-2 and (C) the presence of $\mathrm{W}^{64}$ only on the spike protein of SARS-CoV-2. The black square denotes the amino acids $\mathrm{A}$ and $\mathrm{S}$ at the first small amino acid of the semi-GG4 motif. 
Legends of the Extended Data Figures

324 Extended Data Fig. 1

Dendrogram showing the phylogenetic relationships of the STING and the spike proteins of all

326 coronaviruses based on alignment of amino acid sequences generated by Clustal Omega and hierarchical cluster analysis using $\mathrm{R}$.

Extended Data Fig. 2

330 A motif similarity between the spike protein of SARS-Cov-2 and the TRPM ion channels (TRPM1,TRPM2, TRPM3 and TRPM4) which is very different from the unique motif.

Extended Data Fig. 3

334 Motif similarity (A) for the protein of Mycoplasma pneumonia and the STING proteins, (B) for the proteins of Mycobacterium tuberculosis and the STING proteins and (C) for the proteins of STING 336 proteins and the Klebsialla and Yersinia species.

338 Extended Data Fig. 4

Motif similarity (A) for the C1QT4, beta-lactamase enzymes, STING and the spike protein of

340 SARS-CoV-2, (B) for the beta-lactamase enzymes and C1QT4, spike proteins of SARS-CoV, SARS-CoV-2, ribosomal protein of Methanosprillum hungatei and membrane protein of

342 Methanococcus maripaludis of Archaea and (D) for the spike proteins of SARS-CoV, SARS-CoV2 and beta-lactamase enzymes. There is a new motif similarity different from the unique motif 344 located adjacent to the unique motif. The new motif was shaded and the unique motif adjacent to it was shown by a red square. 


\section{Methods}

The protein data as a compressed single file, named as uniprot_sprot.fasta.gz, was downloaded from

350 the ftp servers of European Bioinformatics Institute (ftp://ftp.ebi.ac.uk). Wget (ver. 1.20.3), gunzip (vers.1.8) and grep (vers.2.25) were used for downloading and extracting protein sequences, Clustal

352 Omega (ver. 1.2.4) for the alignment of proteins ${ }^{51}$, the $\mathrm{R}$ programming language and packages were used for hierarchical cluster analysis, dendrogram and Venn diagrams ${ }^{52}$. Sed (ver. 4.2.2), less (ver.

354 481) and vi improved (vim) (ver.7.4) were used for editing, imagemagic (ver. 6.9.4_9) and enscript (ver.1.6.6) for image editing and producing vector images (pdf). The software listed above were 356 operated under the Slackware GNU/Linux (kernel 4.4.157). The unshaded and unedited results of Clustal Omega were merged into a single pdf file using pdftk (ver. 2.02) and given as supplement.

358 The spike protein of SARS-CoV-2 (PDB ID 6XEY) ${ }^{11}$ for Fig. 4 was created with NGL ${ }^{53}$. The motif similarities in the Figures were shaded. Names of the proteins in the figures are in FASTA style and 360 amino acids are shown as single letter code. 


\section{Data Reporting}

364 Cluster analysis were performed on the proteins for dendrogram and Venn diagrams. No other statistical methods were used in the study. All the protein sequences are deposited on the UniProt

366 servers and the PDB ID of the spike protein of the SARS-CoV-2 used in the study is 6XEY found on the PDB servers.

\section{Author Contributions}

370 Both authors equally contributed to the study.

\section{Declaration of interests}

We declare no competing interests.

374

\section{Additional information}

376 Supplemantary information was given for the unshaded multiple comparison for all figures, as a single pdf file.

378 Correspondence and requests for materials should be addressed to S.A. 
382

384

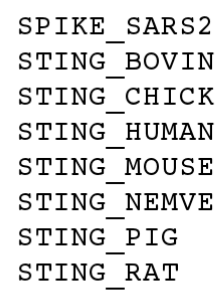

- $\underline{x} \underline{x} \underline{x} \underline{\underline{G}} \underline{y} \underline{L}$

\begin{tabular}{|c|c|}
\hline & \\
\hline GYLI & 186 \\
\hline GYLKVVLPRLK & 191 \\
\hline KGLAPAE I SAVCEKGNFNVA & 186 \\
\hline LGL-----QSLTPAEVSAVCEEKKLNVAHGI & 185 \\
\hline LGL-----RELSKVEE SQLNEKENKNVADGL & 225 \\
\hline LGL-----QHLAPAEVSAICEKRNFNVAHGL & 186 \\
\hline CLDL-----QSLAPAEVSAVCEEKNFNVAHGLAWSYY IGI & 186 \\
\hline & \\
\hline
\end{tabular}

388 Fig. 1:

Alignment of the spike protein of SARS-CoV-2 and the STING proteins. The motif similarity 390 ([AS] $x \times x G Y L)$ is shaded. Black square represents the amino acids Ala and Ser [AS]. 

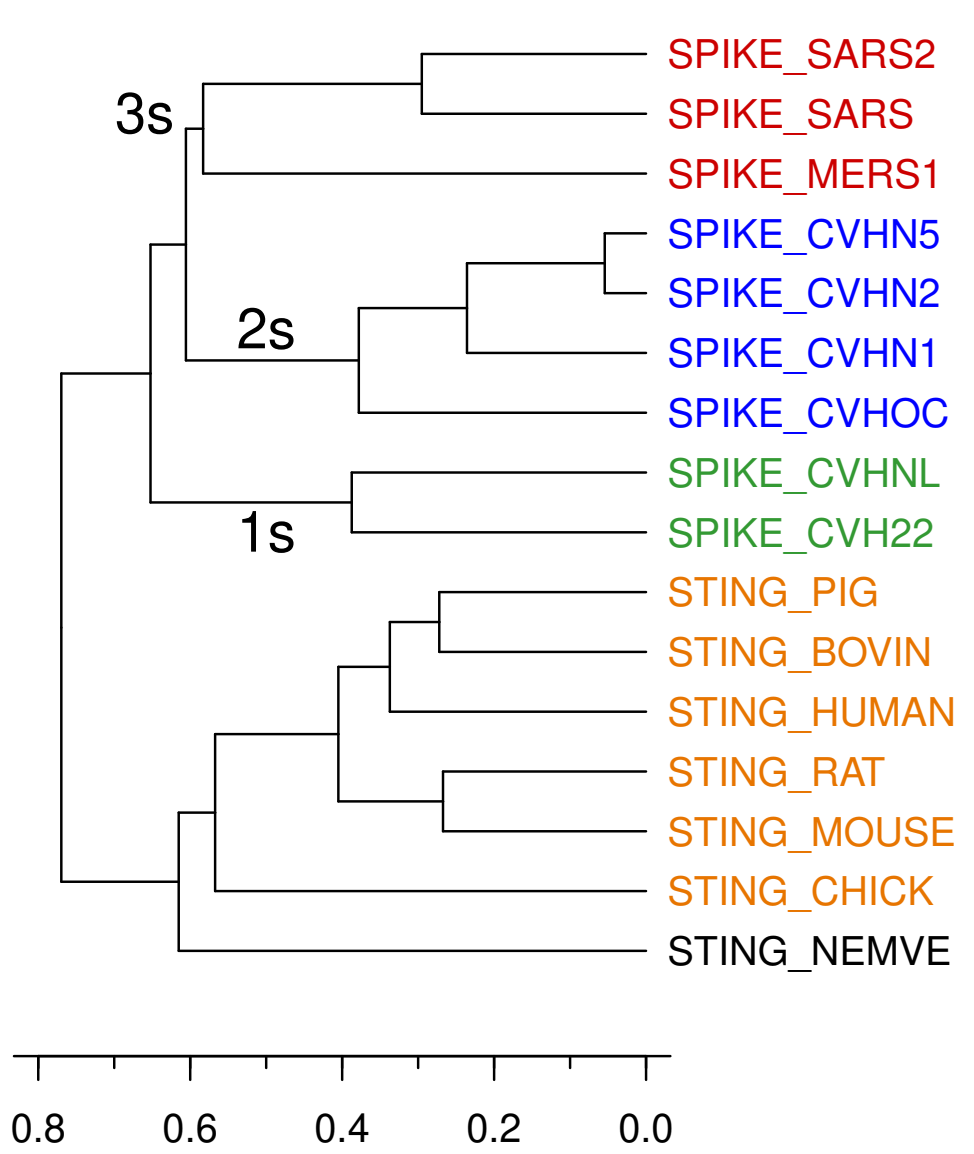

Height
言 $\frac{\widehat{\bar{\sigma}}}{\underline{\underline{\sigma}}}$

$A Y Y V G Y L$

A Y F VGYL

SKRSGFC

LGSSGF L

LGSSGF L

LGSSGF L

LGNLGY L

SNRYGFC

SKRYGFC

SYY IGYL

SYY IGYL

SYY IGYL

SYY IGYL

SYY IGYL

SYY IGYL

SYYFGYL

Fig. 2: Dendrogram of the phylogenetic relationships of the STING and the spike proteins of human

394 beta-coronaviruses based on alignment of amino acid sequences generated by Clustal Omega and hierarchical cluster analysis using R. 'x' denotes any amino acid. The first small amino acid in the small-xxx-small motifs are A, L or S and the other small amino acid is G making the semi-GG4 motif. $1 \mathrm{~s}=$ First group, $2 \mathrm{~s}=$ Second group, $3 \mathrm{~s}=$ Third group. Members of the $3 \mathrm{~s}$ are the cause of viral outbreaks of the $21^{\text {st }}$ century and the ongoing pandemic of COVID-19. 


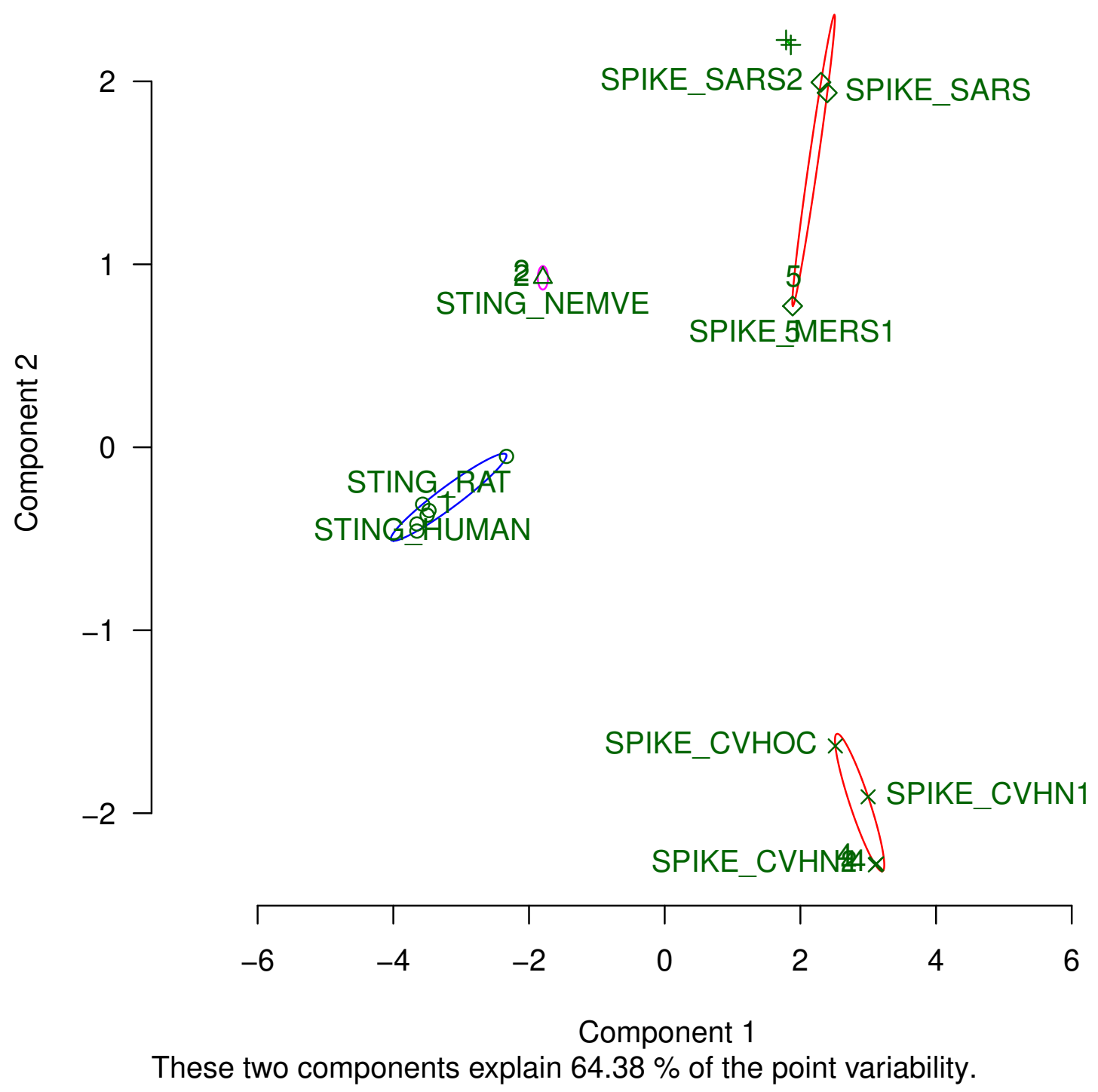

Fig. 3: Venn diagram of the STING and the spike proteins of human beta-coronaviruses showing 404 the $3 \mathrm{~s}$ as a distinct group as if it is evolving towards a new group of beta-coronavirus. The same STING and the spike proteins shown in Fig. 2 were used for this Venn diagram. 
(A)
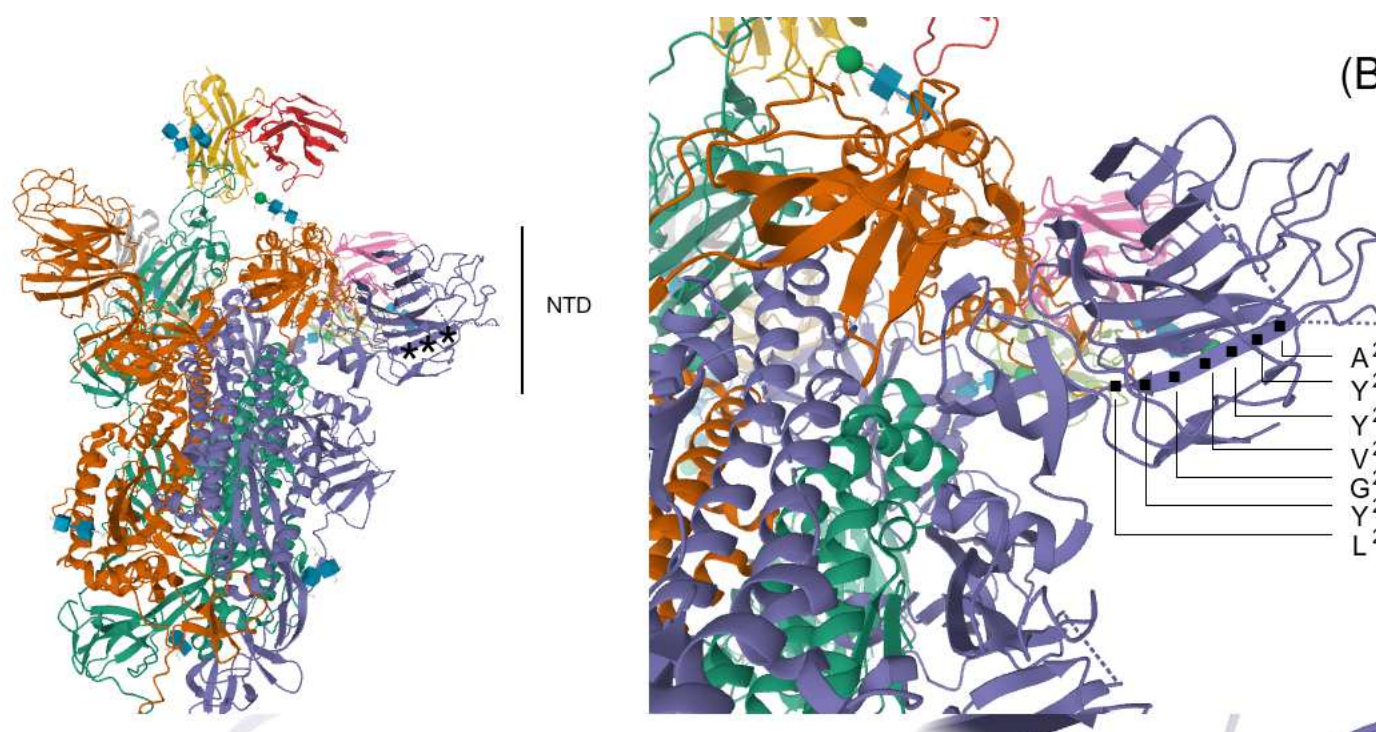

(B)

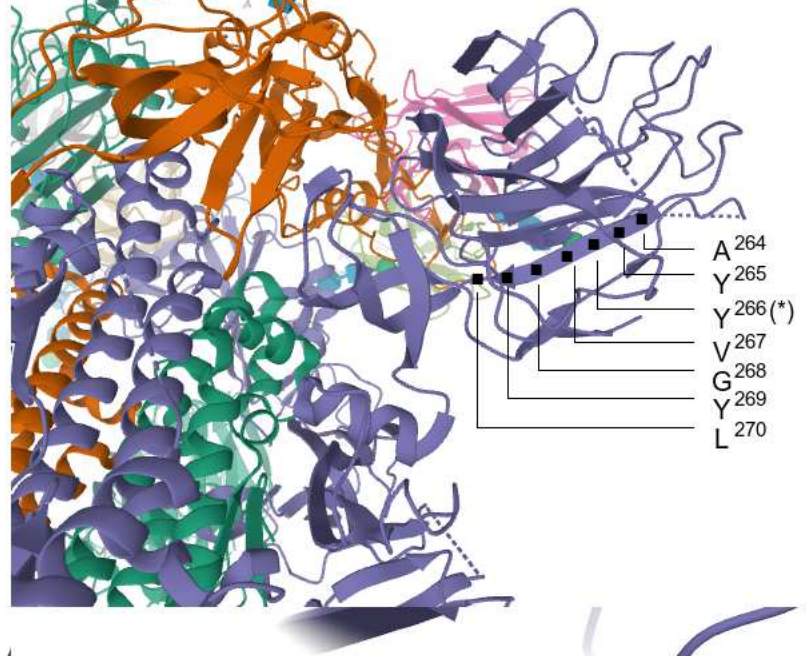

(C)

1
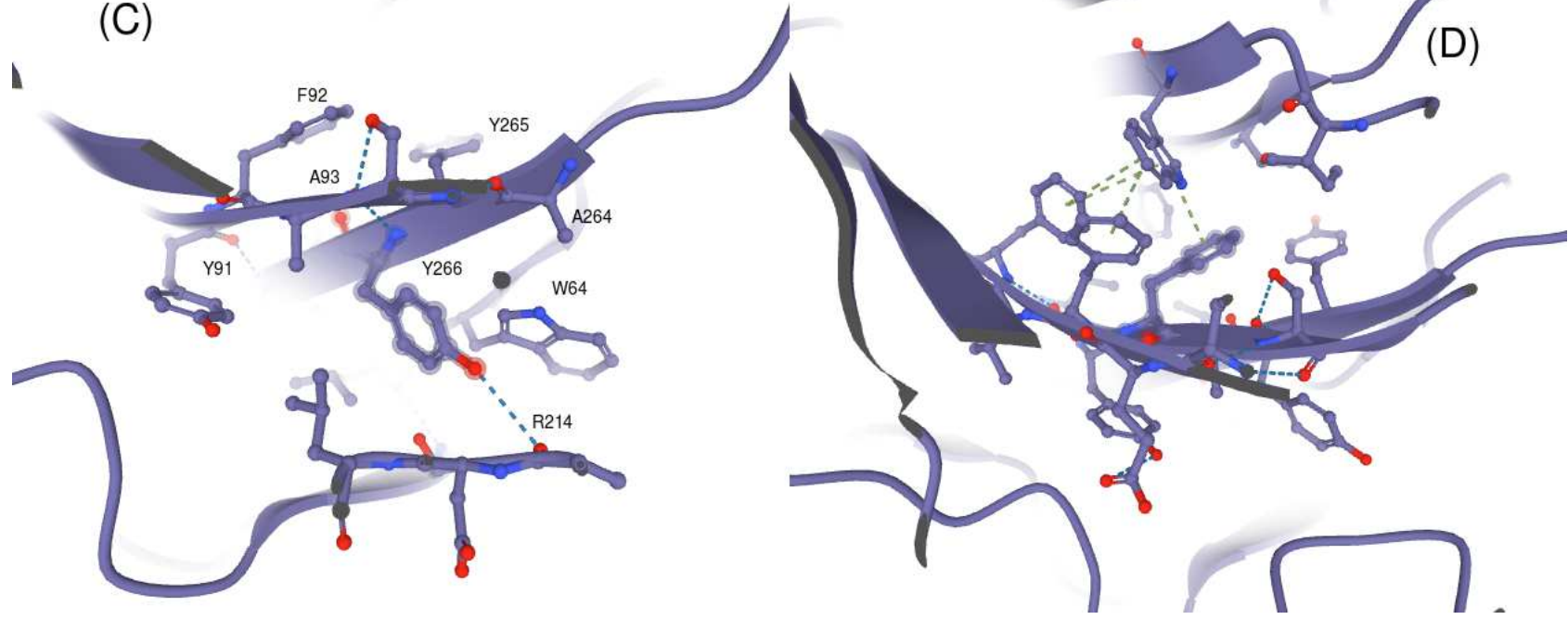

410

Fig. 4: The unique motif is, (A) on the NTD of the spike protein marked with stars, (B) located on

412 one of the beta-sheets with a finger like loop extending outside, (C) there is a hydrogen bond between the $\mathrm{R}^{214}$ of the neighbour beta-sheet and $\mathrm{Y}^{266}$ which is found only on the SARS-CoV-2

414 member of the 3s group. $\mathrm{Y}^{266}$ makes an aromatic cage with the $\mathrm{W}^{64}$ which is found only on SARSCoV-2, (D) another aromatic cage around $\mathrm{A}^{93}$, the unique motif is surrounded with aromatic cages. 
(A)

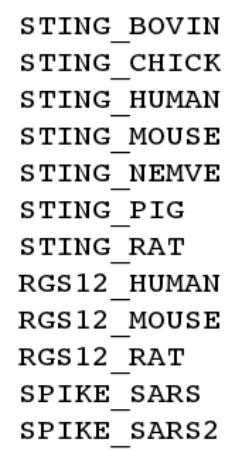

(B)

$$
\begin{aligned}
& \text { RGS 12_HUMAN } \\
& \text { RGS 12_MOUSE } \\
& \text { RGS12_RAT } \\
& \text { SPIKE_SARS2 }
\end{aligned}
$$

(C)

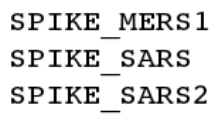

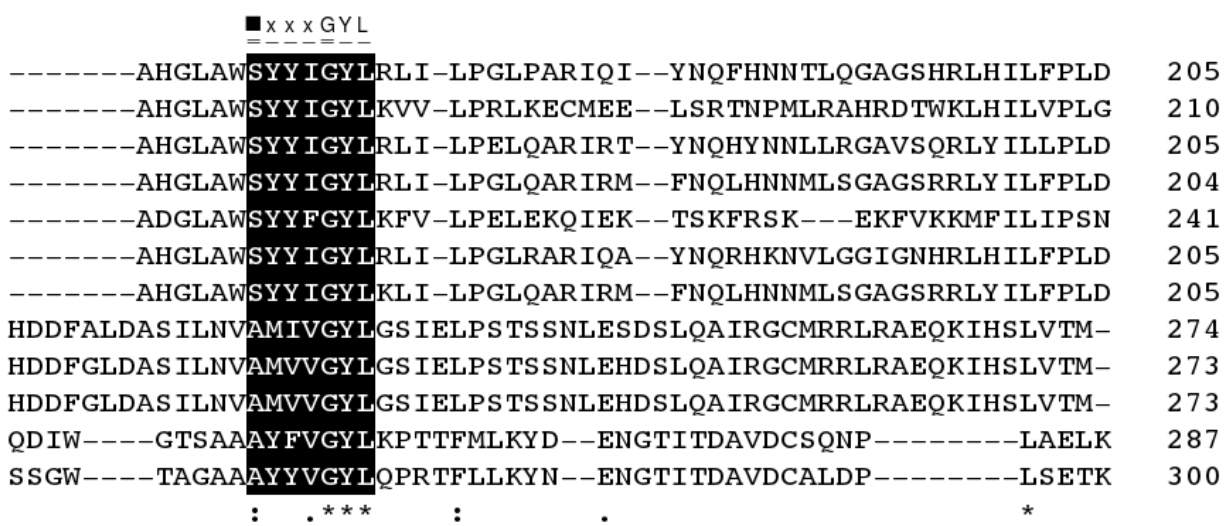

$\begin{array}{cc}\text { A } \times x \times G Y L & \\ \text { SKVIHDDSVFS IGLESHDDFALDAS ILNVAMIVGYLGS IELPS TS SNLESDSLQAIRGCM } & 259 \\ \text { SKVINDDSVF TVGLDSHDDFGLDAS ILNVAMVVGYLGS IELPS TS SNLEHDSLQA IRGCM } & 258 \\ \text { SKVINDDSVF TVGLDNHDDFGLDAS ILNVAMVVGYLGS IELPS TS SNLEHDSLQAIRGCM } & 258 \\ \text { QTLLALHRSYLTPGDS S GWTA----GAAAYYVGYLOPRTFLLKY --NENGTITDAVDCA } & 292\end{array}$
..: . : : : . . :

QGRTY SNITITY QGLF-PYQGDHGDMYVY SAGHATGTTPOKLFVANY SODVKOFANGFVV 118 DEIFRSDTLYLTODLFLPFY SNVT---GFHTIN-------HT----FGNPVIPFKDGIYF 89 DKVFRSSVLHS TQDLFLPFFSNVT---WFHAIHVSGTNGTKR----FDNPVLPFNDGVYF 92

422 Fig. 5: Motif similarity (A) for the STING, RGS12 and the spike proteins of SARS-CoV and SARS-CoV-2, (B) for the RGS12 and the spike protein of SARS-CoV-2 and (C) the presence of $424 \mathrm{~W}^{64}$ only on the spike protein of SARS-CoV-2. The black square denotes the amino acids A and S at the first small amino acid of the semi-GG4 motif. 


\section{Figures}

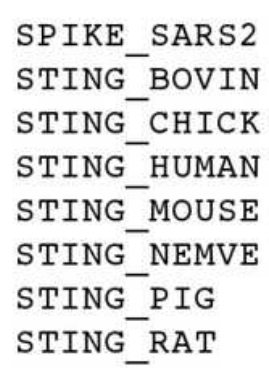

- $\underline{x} \underline{x} \underline{x} \underline{G} \underline{\underline{Y}} \underline{\underline{L}}$

\begin{tabular}{|c|c|c|}
\hline INITRFOTLLALHRSYLTPGDSSSGWTAG & CFLL & 281 \\
\hline ILLGL-----QGLAPAEVSAICEKRNFNVAHGL & SYY IGYLRLILPGLPAR IQ IYNQFH & 186 \\
\hline ALGL-----QKLSAVEVSELTESSKKNVAHGL & SYY IGYLKVVLPRLKECMEELSRTN & 191 \\
\hline ILLGL-----KGLAPAE I SAVCEKGNFNVAHGI & SYY IGYLRL ILPELQAR IRTYNQHY & 186 \\
\hline MLLGL-----QSLTPAEVSAVCEEKKLNVAHGL & SYY IGYLRL ILPGLQAR IRMFNQLH & 185 \\
\hline HLLGL-----RELSKVEE SOLNEKENKNVADG] & SYYFGYLKFVLPELEKQIEKTSKFR & 225 \\
\hline ILLGL-----QHLAPAEVSAICEKRNFNVAHGL & SYY IGYLRLILPGLRAR IQAYNQRH & 186 \\
\hline MTLDL-----QSLAPAEVSAVCEEKNFNVAHGL & KLILPGLQARIRMFNQLH & 186 \\
\hline$: .:$ & * & \\
\hline
\end{tabular}

\section{Figure 1}

Alignment of the spike protein of SARS-CoV-2 and the STING proteins. The motif similarity ([AS]xxxGYL) is shaded. Black square represents the amino acids Ala and Ser [AS]. 


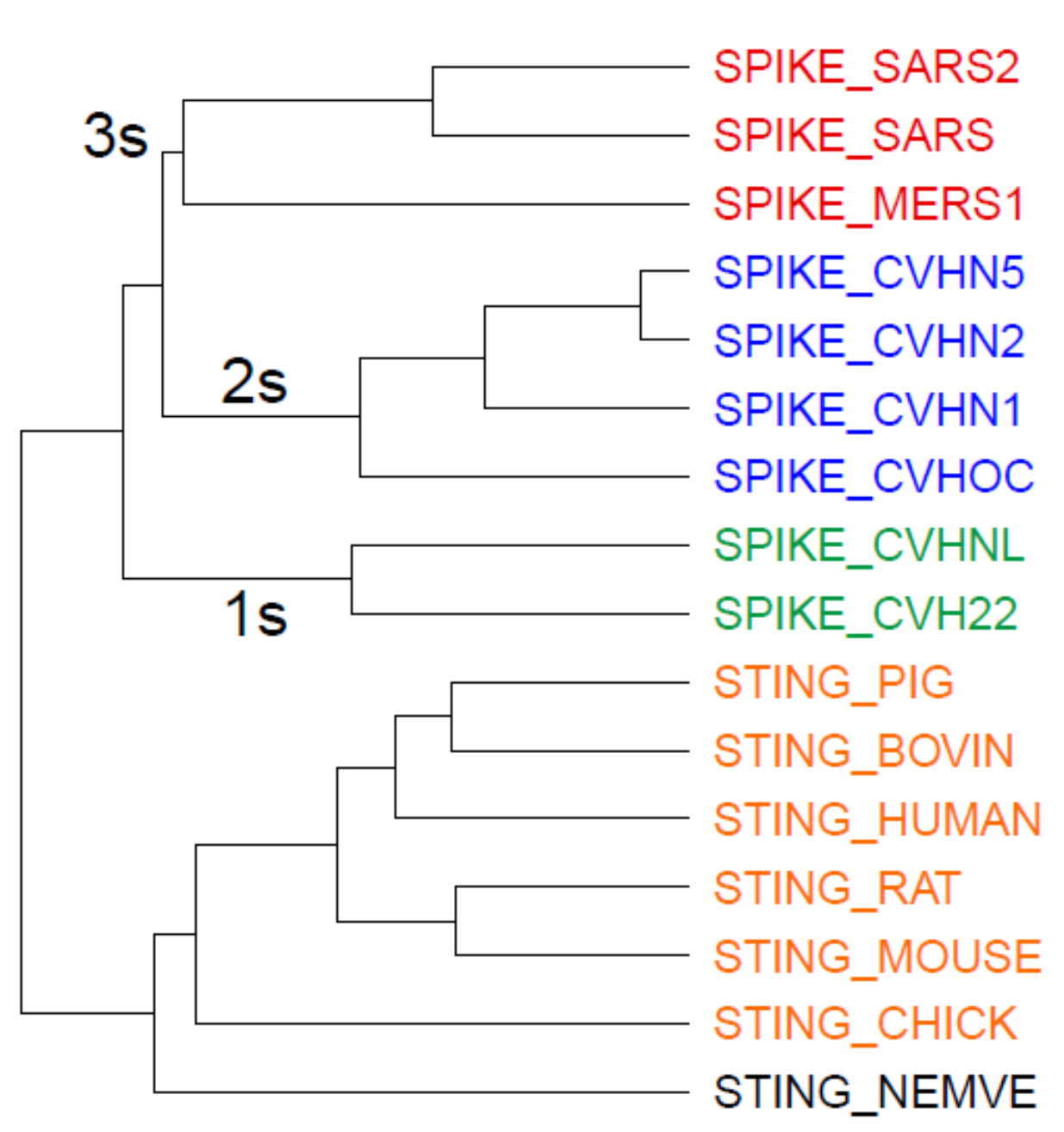

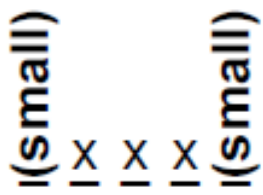

A Y Y V G Y L

A Y F VGYL

SKRSGFC

L GS S F L

L GSSGF L

L GSSGF L

L GN L G Y L

S NRYGF C

SKRYGF C

$S Y Y \mid G Y L$

$S Y Y \mid G Y L$

S Y Y I GYL

$S Y Y \mid G Y L$

$S Y Y \mid G Y L$

$S Y Y \mid G Y L$

$S Y Y F G Y L$

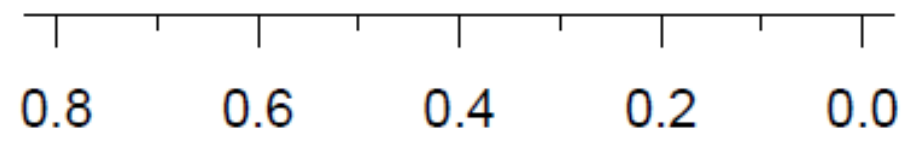

Height

Figure 2

Dendrogram of the phylogenetic relationships of the STING and the spike proteins of human betacoronaviruses based on alignment of amino acid sequences generated by Clustal Omega and hierarchical cluster analysis using R. ' $x$ ' denotes any amino acid. The first small amino acid in the smallxxx-small motifs are A, $\mathrm{L}$ or $\mathrm{S}$ and the other small amino acid is $\mathrm{G}$ making the semi-GG4 motif. $1 \mathrm{~s}=$ First group, $2 \mathrm{~s}=$ Second group, $3 \mathrm{~s}=$ Third group. Members of the $3 \mathrm{~s}$ are the cause of viral outbreaks of the 21st century and the ongoing pandemic of COVID-19. 


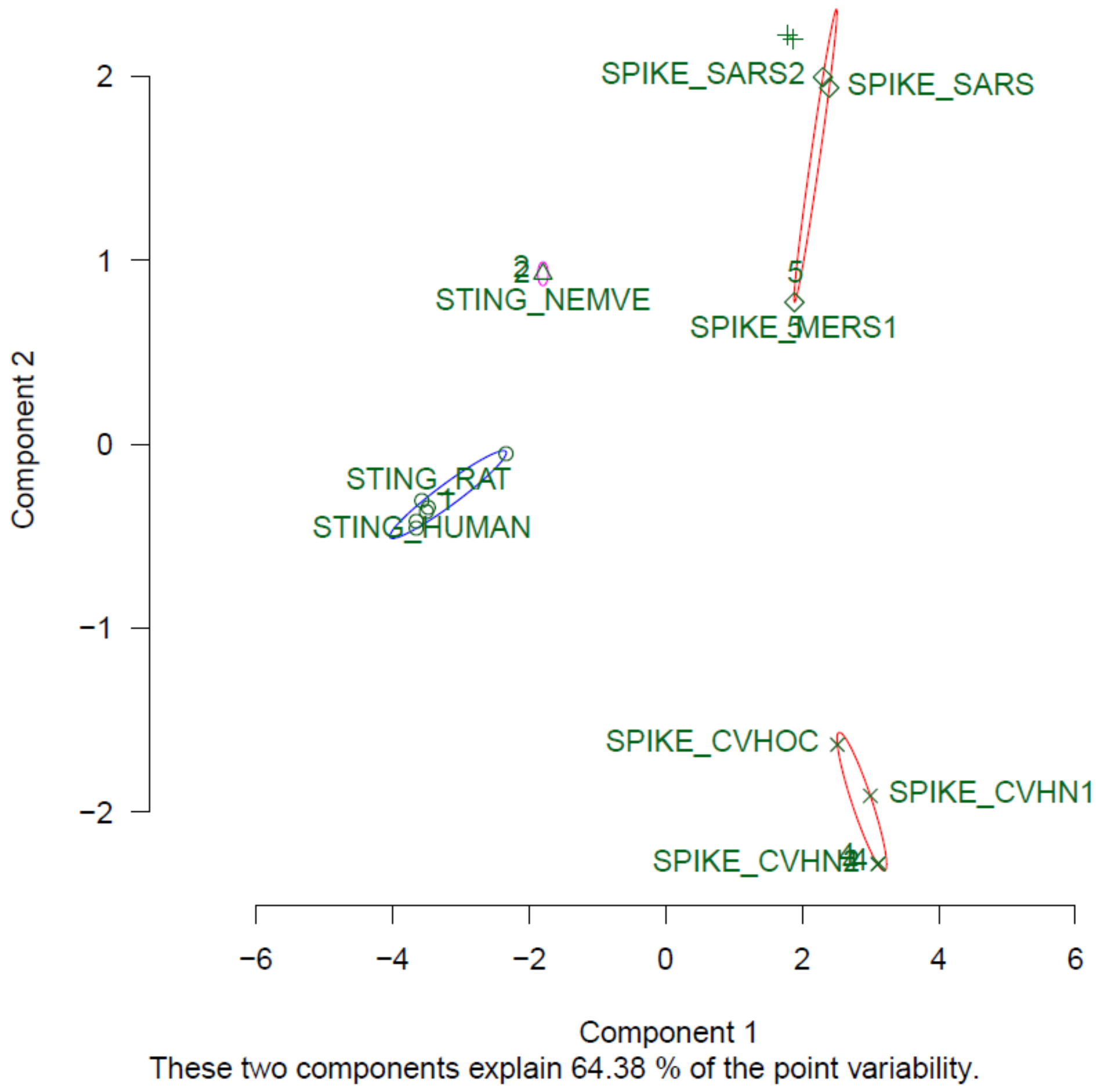

Figure 3

Venn diagram of the STING and the spike proteins of human beta-coronaviruses showing the $3 \mathrm{~s}$ as a distinct group as if it is evolving towards a new group of beta-coronavirus. The same STING and the spike proteins shown in Fig. 2 were used for this Venn diagram. 
(A)
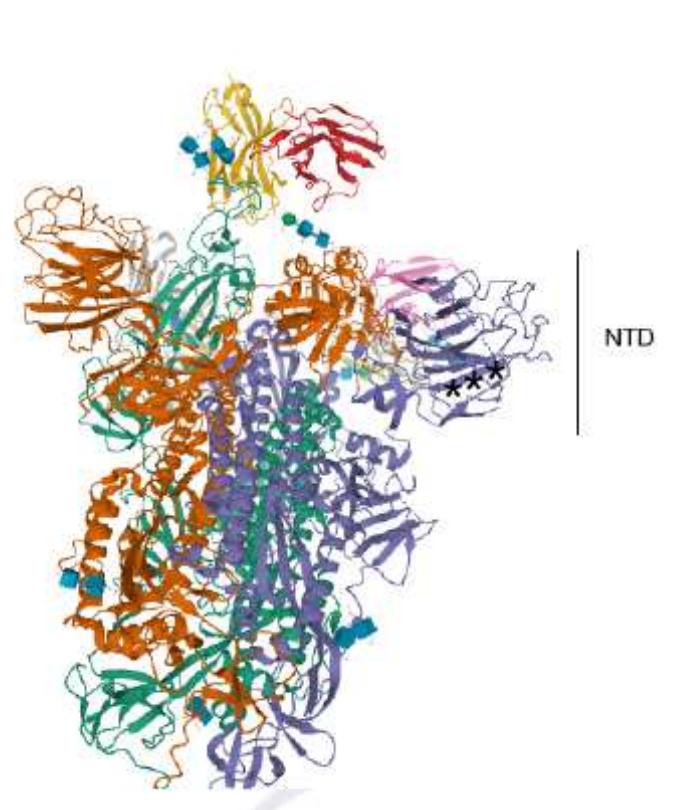
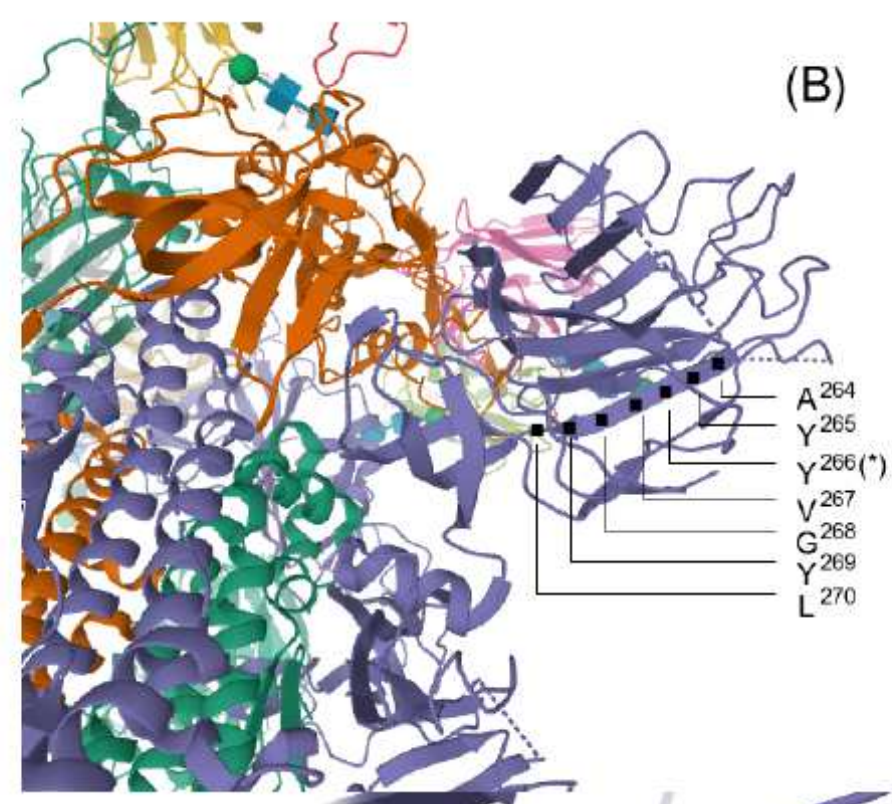

(C)

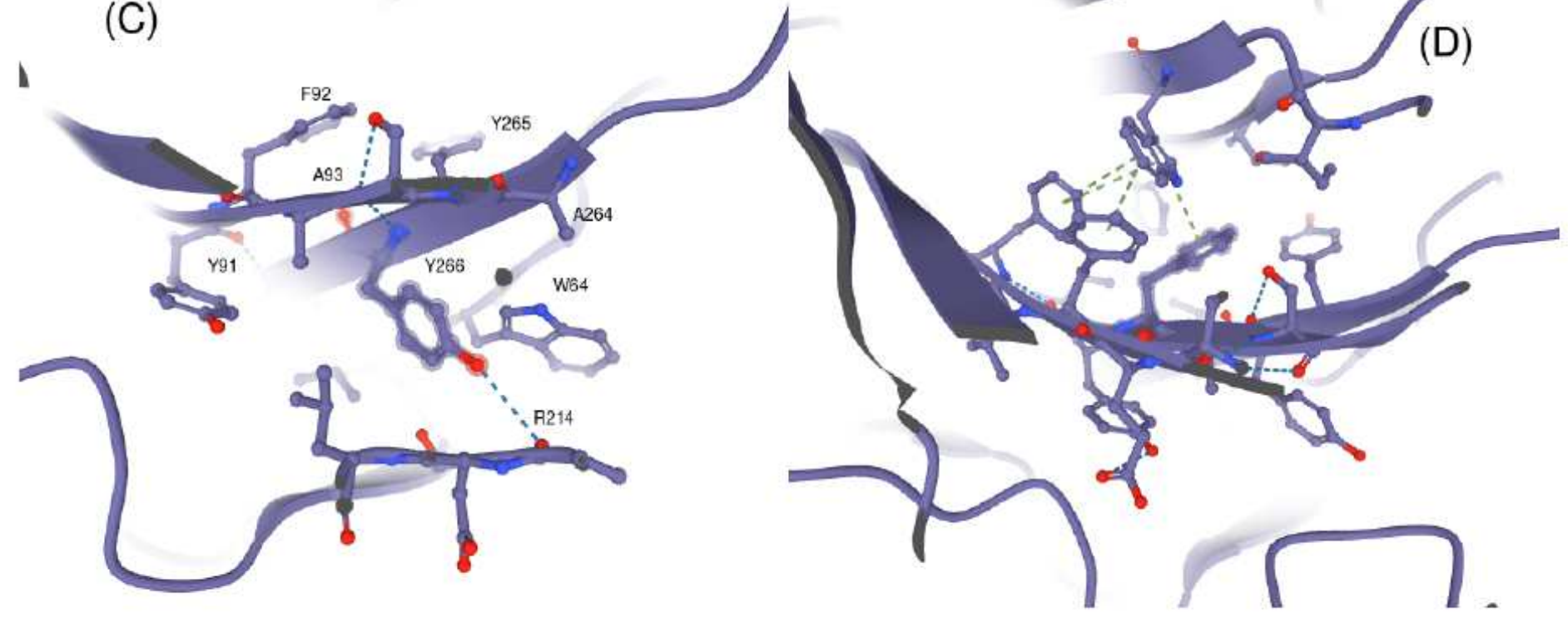

Figure 4

The unique motif is, (A) on the NTD of the spike protein marked with stars, (B) located on one of the betasheets with a finger like loop extending outside, (C) there is a hydrogen bond between the R214 of the neighbour beta-sheet and Y266 which is found only on the SARS-CoV-2 member of the 3s group.Y266 makes an aromatic cage with the W64 which is found only on SARSCoV-2, (D) another aromatic cage around $A 93$, the unique motif is surrounded with aromatic cages. 
(A)

STING_BOVIN STING CHICK STING_HUMAN STING MOUSE STING_NEMVE STING PIG STING RAT RGS 12 HUMAN RGS12_MOUSE RGS12 RAT SPIKE_SARS SPIKE_SARS2

(B)

RGS 12 HUMAN RGS 12 MOUSE RGS 12 RAT SPIKE_SARS 2

(C)

SPIKE MERS 1 SPIKE SARS SPIKE_SARS 2

\begin{tabular}{|c|c|c|}
\hline FIt & QI--YNQFHNNTLQG & 205 \\
\hline------ AHGL & YY IGY LKVV-LPRLKECMEE--LSR TNPMLRAHRD TWKLH ILVPLG & 210 \\
\hline----- AHGLA & VSYY IGYLRLI-LPELQARIR T--YNQHY NNLLRGAVS QRLY ILLPLD & 205 \\
\hline-- AHGL & YLRLI-LPGLQARIRM--FNQLHNNMLS GAGSRRLY ILFPLD & 204 \\
\hline--- ADGLA & VSYYFGY LKFV-LPELEKOIEK--TSKFRSK---EKFVKKMF IL IPSN & 241 \\
\hline---- AHGLA & NSYY I GY LRLI-LPGLRAR IQA - -YNQRH KNVLGG I GNHRLH ILF PLD & 205 \\
\hline----- AHGLAW & NSYY IGY LKLI-LPGLQARIRM - -FNQLHNNMLS GAGSRRLY ILFPLD & 205 \\
\hline DDFALDAS ILI & SIELPS TSSNLESDSLQA IRGCMRRLRAEOKIHSLVTM - & 274 \\
\hline HDDFGLDAS ILI & JAMVVGY LGS IELPS TSSNLEHDSLQA IRGCMRRLRAEOKIHSLVTM- & 273 \\
\hline HDDFGLDAS ILI & VAMVVGY LGS IELP S TS SNLEHD SLQA IRGCMRRLRAEQKIHSLVTM- & 273 \\
\hline QDIW----GTSAA & 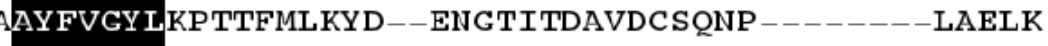 & 287 \\
\hline SGW----TAGAA & YYVGYLQPRTFLLKYN--EINGTITDAVDCALDP--------LSETK & 300 \\
\hline
\end{tabular}

A $\times \times \times G Y L$

S KVIHDDSVFS IGLES HDDFALDAS ILNVAMIVGY LGS IELPS TS SNLESDSLQAIRGCM 259 SKVINDD SVFTVGLD SHDDFGLDAS ILNVAMVVGY LGS IELPS TS SNLEHD SLOAIRGCM 258 S KVINDD SVFTVGLDNHDDFGLDAS ILNVAMVVGY LGS IELPS TS SNLEHD SLOAIRGCM 258 QTLLALHRSYLTPGDS S SGWTA----GAAAYYVGY LOPRTFLLKY --NENGTITDAVDCA 292

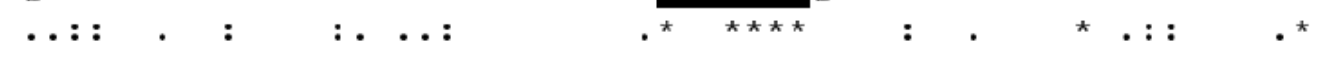

QGR TY SNITI TYQGLF-PYQGDHGDMYVY SAGHATGTTPOKLFVANY SODVKOFANGFVV 118 DEIFRSDTLYLTQDLFLPFY SNVT---GFHTIN-_-_---HT-_--FGNPVIPFKDGIYF 89 DKVFRS SVLHSTQDLFLPFFSNVT---WFFHA IHVSGTIJGTKR----FDNPVLPFNDGVYF 92

\section{Figure 5}

Motif similarity (A) for the STING, RGS12 and the spike proteins of SARS-CoV and SARS-CoV-2, (B) for the RGS12 and the spike protein of SARS-CoV-2 and (C) the presence of W64 only on the spike protein of SARS-CoV-2. The black square denotes the amino acids $A$ and $S$ at the first small amino acid of the semiGG4 motif.

\section{Supplementary Files}

This is a list of supplementary files associated with this preprint. Click to download.

- extendeddatafig01.pdf

- extendeddatafig02.pdf

- extendeddatafig03.pdf

- extendeddatafig04.pdf

- supplementforfigures.pdf 